\title{
Proposal of the Promotional Video Design Model of Culture Contents, using 3D Image Moving Technique: Applying the Augmented Reality and E-museum Concepts
}

\author{
Ga Ram Choi ${ }^{1}$ and Hak Hyun Choi ${ }^{1}$ \\ 1 Department of Contents Design, Seoul Women's University, 621 Hwarang-ro, \\ Nowon-gu, Seoul, 139-774, Korea \\ grchoi@dclab.kr, choiidea@gmail.com
}

\begin{abstract}
This study is about the new expressive techniques for promotional videos for culture contents and aims to propose a new design model for making effective prontotional videos using augmented reality technology and E-museum concepts. Augmented reality is one of the up-rising intelligent information technology, but has been barely used for promotional videos. However the technology enables the interaction between physicat objects in the real world and virtual image though digital devices. Augnented reality opens the door for connecting real and virtual world, and invites humans to the virtual environment. There are growing numbers attempt to move off-line culture contents into on-line space. Considering the connection between culture contents and digitat environment, more studies about the convergence is necessary in the cultural-art industry, especially for marketing business. Therefore, this study aims to take a brief look ataugmented reality technology, and proposes the new experimental modet and expressive techniques for cultural-art organizations' promotional video, which set the experientiat learning environment, and enables consumers to take contents actively.
\end{abstract}

Keywords: Augmented Reality, Lser Interaction, Digital Overlay, E-museum

\section{Introduction}

Recently, most of cultural organizations, such as museums or art galleries and companies have been applied Information \& Communication on their systems. However, the range of the convergence covers bulding information database or early virtual reproducing. There have been attempts to combine augmented reality or virtual reality technologies to serve differentiated experience or build virtual environments. However, Most of the organizations are using ICT for image, or video processing. For example, mobile applications of one of the major museums of South Korea, National Museum of Korea, 'National Museum of Korea' and 'Smart Curator' imitate digital curation service by serving audio curation service via mobile applications. If we take a look at the contents it the apps, we can see most of the contents consist of simple images, video clips or audio combined information. On the other hands, the mobile heritage tour applications of Cultural Heritage Administration seem to serve interesting, 3dimentional information and mobile tour systems. Those apps are based on GPS and Augmented reality technologies. User downloads the apps on their mobile device or tablet and activates GPS, that way the apps know where the user located. The user operates the system by taking pictures of some of heritages and getting 3D images or virtual information from the applications. Augmented reality is used for showing images of the heritage in the past, or heritages don't exist anymore. Those apps also enables user to see 
virtual images of flowers or butterflies on the wall using media-façade technique. Overall, the convergence of ICT and cultural-art contents gives user better experience and makes them interact with objects though the technology. However those advanced techniques are applied on just few of the cultural contents, and there are still lost of cultural contents seating on the simple technology. Considering the culture contents industry has been developed as the economical environment improved, and latent demand for culture contents is expected to be pretty high, studying Information \& Communication Technology and finding effective ways to combine it with culture contents are certainly necessary.

\section{Related Works}

\subsection{Mobile Application}

Here is an example of AR based mobile applications designed by Cultural Heritage Administration. This handy app consists of Augmented Reality, 3D imaging Meda-Façade techniques. User carries the app and explores and interacts with outdoo heritage, Gyongbokgung Palace of Seoul in case of this app. The augmented realit based app contains 2dimentional information, such as the cultural assets information photo gallery, or exhibiting information, or 3 dimentional user-centered experience at the same time. There are 3 concepts setting for enjoying Gyongbokgung Palace, which are regular, time-traveling, and character experience settings. User chooses one of the settings and experiences the heritage with special point of view. There are noticeable ICT features in the app below

\subsubsection{GPS based Service}
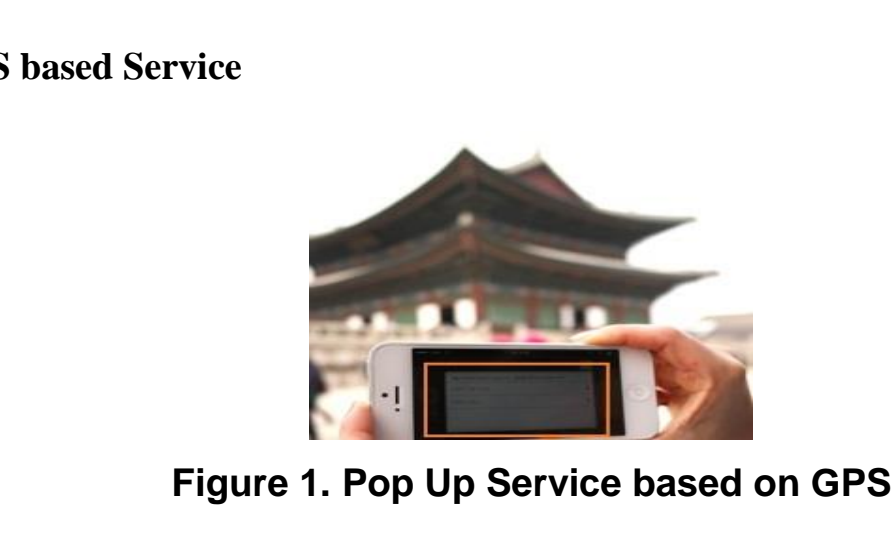

The system sees where the user located in using Global Positioning System, and shows the viewpoints or cultural spots information using Pop-up. When user get to certain area set by the system, infornation automatically pops up and asks if they want to see or hear more. In addition, GPS based map shown on Figure 3 marks user's location in real time on the map, and help them find out where they are located in by scanning objects around the users.
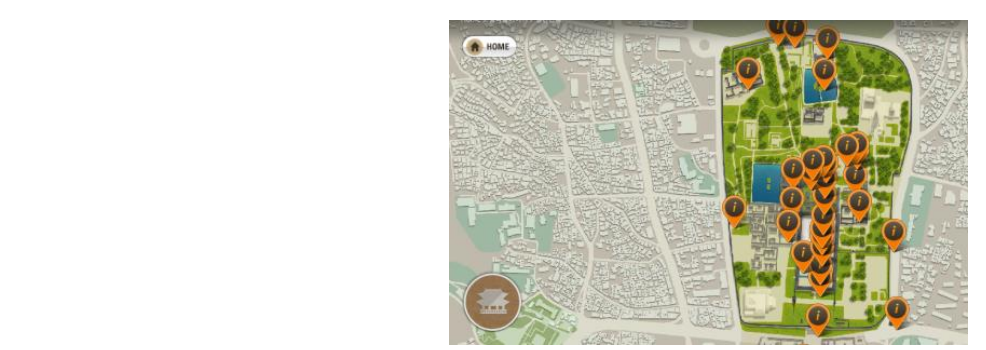

Figure 2. GPS based Map Service 
2.1.2. Augmented Reality: The very fascinating point of the app is that this app scans objects or buildings, and overlays 3D pictures of the past or shows a reconstruction of buildings which is not exist anymore. This technique is based on augmented reality. User focuses their mobile device on the objects, fits them in the line shown on the screen, then the augmented reality system recognizes them and calls saved $3 \mathrm{~d}$ images on the screen. That enables user to see things are not in the real world, along with seeing things of the real world, which broadens user experience by giving double-way interaction.

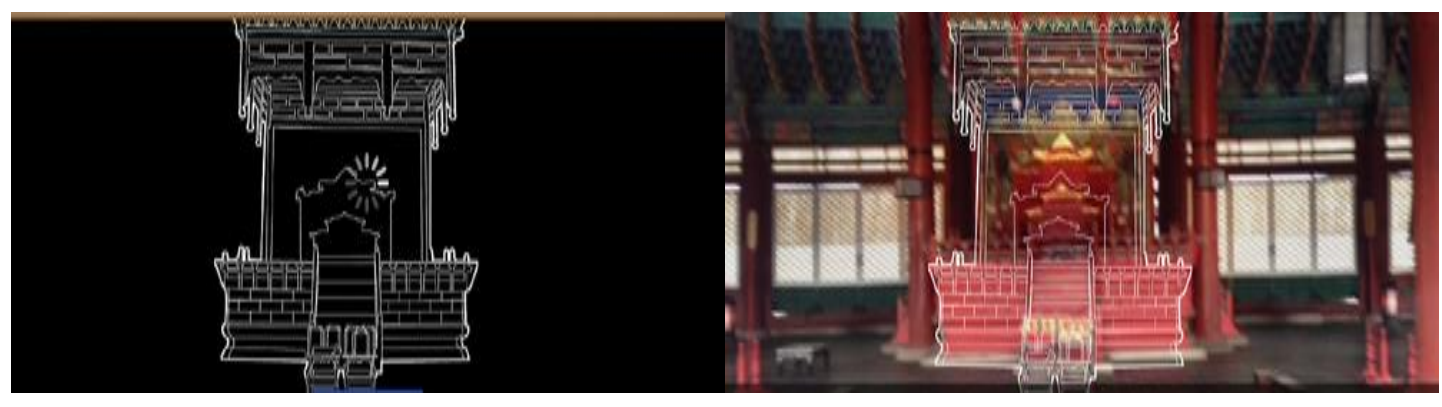

Figure 3. Delivering Inexistent Images using Augmented Reality

2.1.3. 3D Imaging Techniques: In the different version of the app, Deoksugung Palace, serves the reproduction of important buildings or other cultuassets. The buildings seen in figure 5 are not exist anymore, but the reprodaced 3D images feature thought the app and user sees what the building was look like, or what was in the empty spot.

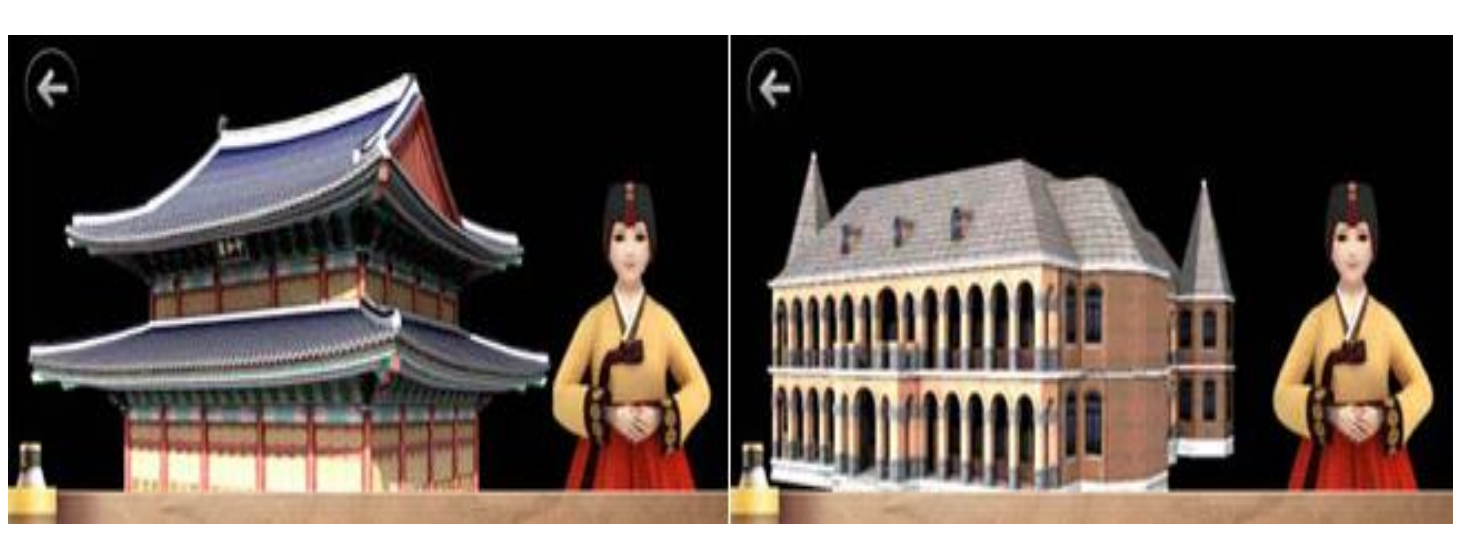

Figure 4. Reproduced 3D Images of Buildings

\subsection{Analysis of Augmented Reality}

2.2.1. Augmented Reality: Augmented Reality (AR) is technology showing virtual images on the top of real image or background, which is mainly focusing on increasing the interaction between human and computer using 3D processed graphic [1]. Augmented Reality is called Mixed Reality since AR is adding virtual information on the real environment, which is different from virtual reality.Augmented Reality technology introduced in Communications of the ACM의 special issue (July 1993) for the first time [2]. Since the special issue published, there have been lots of researches about augmented reality and the approach. In sum, Augmented Reality opens possibility of using real objects as usual, but enables connection to computer networks using them [9]. 


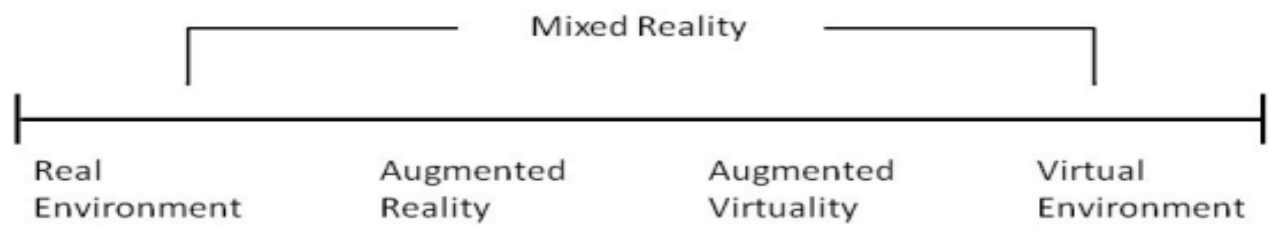

Figure 5. Milgram's Reality-virtuality Continuum [3]

In 1994, Paul Milgram and Fumio Kishino defined 'reality-virtuality continuum'. As shown on figure 7, Augmented Virtualityis located between Real Environment and Virtual Environment.In 1997 and 2007, Ronald Azuma introduced his research about Augmented reality $[4,5]$, and defined augmented reality as 3 features below.

- Augmented Reality is mixing the real and virtual world

- Augmented Reality interacts in the real world

- Augmented Reality works based on the 3D technology

In 2002, Mann [6] edited on another axis on the Milgram's seality-virtuality continuum, Mediality (left). The right van diagram of figure 6 defines Mediated reality and Mediated virtuality. In Mediated reality, The system changes the real world by mixing (augmented reality), diminishing (diminished reality), and by modulating (modulated reality). By diminishing existing elements or objects of the real world, the real world can be mediated in a different way from virtual reality [7].

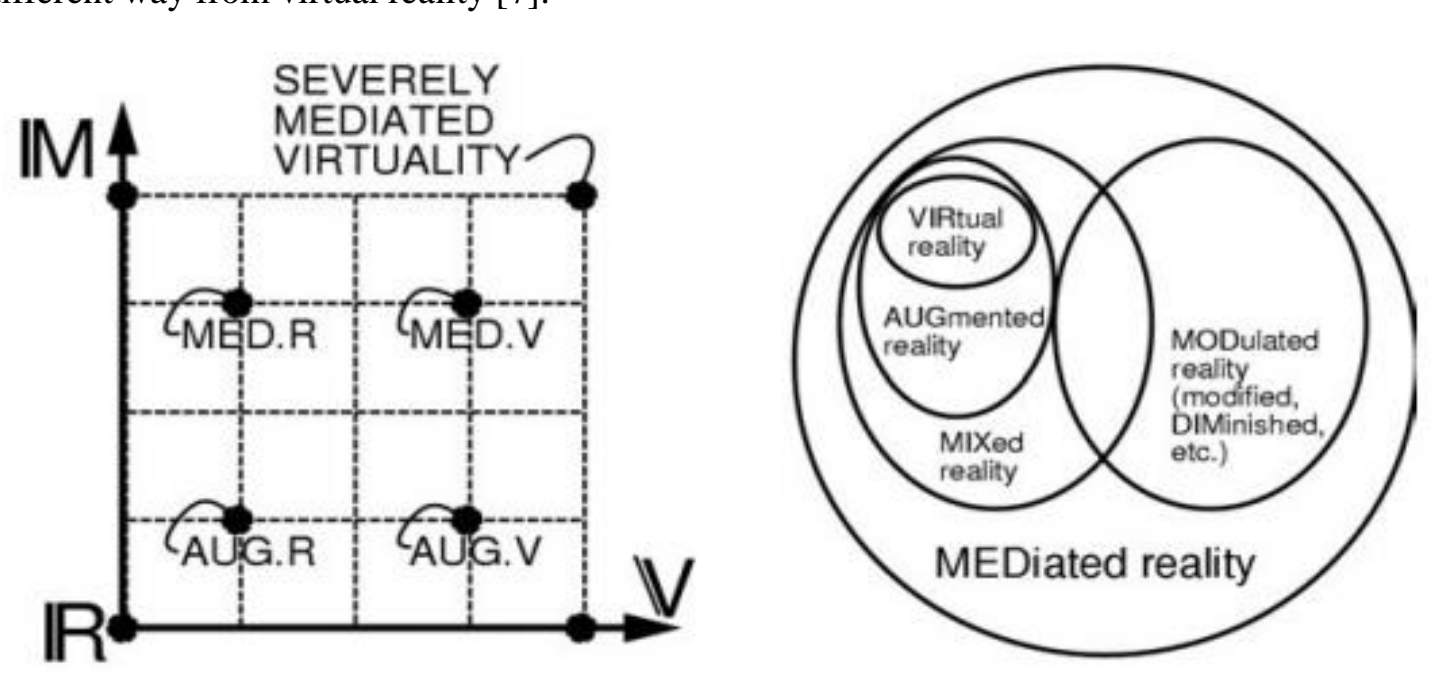

Figure 6. Mann's Reality-virtuality-mediality Continuum from [6]

2.2.2. The Basic Principle of Augmented Reality: There are complex techniques for Augmented Reality, users, physical objects, and the environment. The user holds devices on their hands to approach to information of the objects [2]. In order to realize Augmented Reality, GPS system or gravitational sensor (or gyroscope sensor), positioning system are needed. The positioning system gets angle, location information and puts that into Augmented Reality applications. Augmented Reality system takes information, processes it, and prints out the output, which is the final images of information mapping, through Smartphone or Tablet PC. 
Table 1. Example of Augmented Reality Approach [2]

\begin{tabular}{|llll|}
\hline Augment: & Approach & Technology & Applications \\
\hline Users & Wear devices & VR helmets & Medicine \\
& on the body & Goggles & Field service \\
& & Data gloves & Presentations \\
\hline Physical objects & Imbed devices & Intelligent bricks & Education \\
& within objects & Sensors, receptors & Office facilities \\
& & GPS, electronic paper & Positioning \\
\hline Environment & Project images and & Video cameras, Scanners & Office work \\
surrounding objects & record remotely & Graphics tablets & Film-making \\
and users & & Bar code readers & Construction \\
& & Video Projectors & Architecture \\
\hline
\end{tabular}

Table 1 above is the approach to 3 elements of Augmented Reality, which are users, Physical objects, Environment surrounding objects and lisers Each approach has pros and cons and can be choose as the purpose of use. The inportant point is clearly defining how to make objects and humans interact, identifying additional computing problems and technically developing right solutions [2].

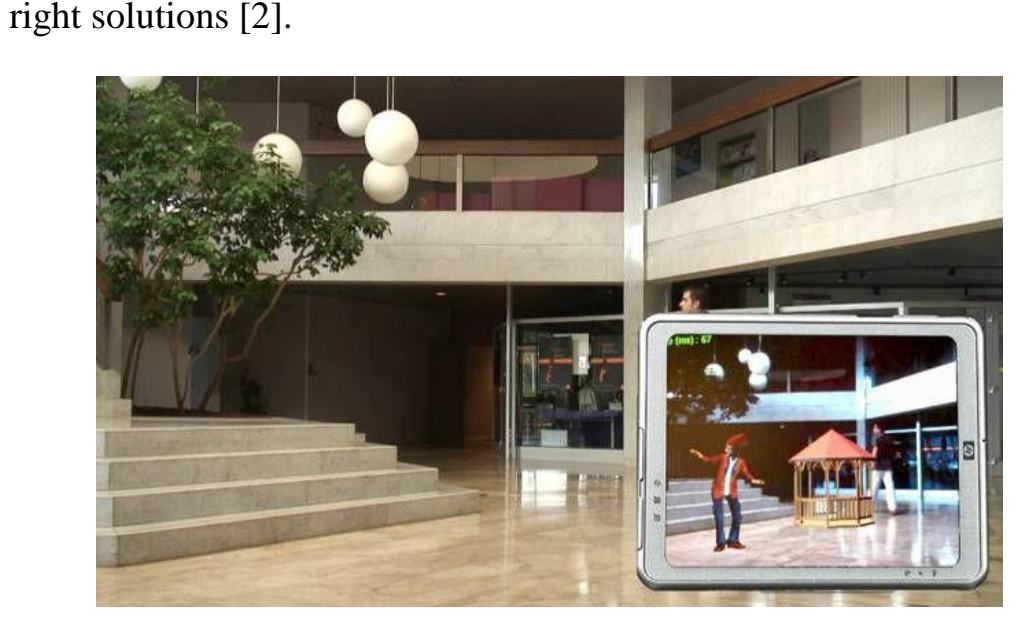

Figure 7. Augmenting the Real World with Digital Overlays [10]

\subsubsection{Status of Utilizing Augmented Reality}

Augmented Reality technology is being realized in a diverse of platforms such smartphone, tablet, Kiosks, web, which runs applications and camera. Augmented Reality market contains 5 kinds or markets using the technology. Smartphone, Eyewear, Tablet, Head mounted displays, and Head up displays. There are some advanced industry markets as well, such as, National defense, Medical, Manufacturing, and Repairing. Business market, consumption market in other worlds, consists of Game, E-learning, GPS, or online advertisement. Also, there are numbers of augmented reality design companies such as Total Immersion (France), Metaio GmbH (Germany), Layar (Netherlands), Wikitude (Austria) [3]. Table 2 is the Predicted market size of Augmented Reality from 2011 to 2016, analyzed by Marketsandmarkets. As shown below, the predicted size of augmented reality market is gradually growing. 


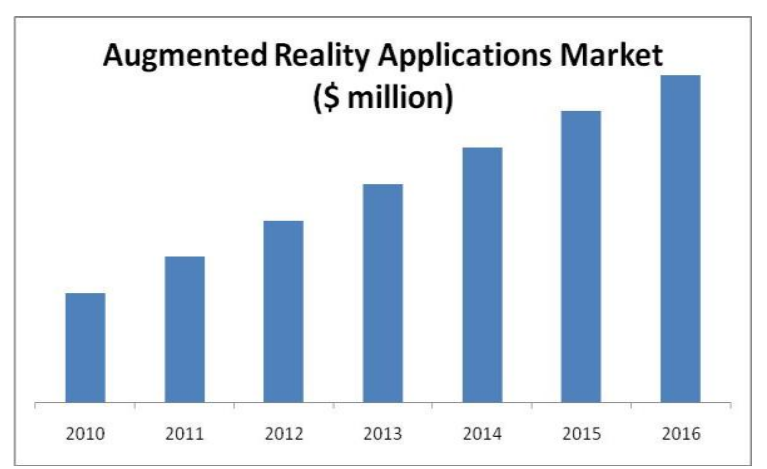

Figure 8. Predicted Market Size of Augmented Reality (2011-2016) [8]

\section{Mobile Augmented Reality (Analysis of Existing Promotional Videos)}

Figure 8 below is a sample image of the augmented reality app and magazne service produced by GQ, a global magazine company. Basically, the core point is connecting user and the magazine using 'GQ Live!' augmented reality applications. There are over 270 augmented reality motions in the 160 pages magane which can be aetivated though the mobile applications.

\subsection{Promotional video A}

Promotional video A, made and released in 2013, is designed to promote National Museum of Korea, South Korea and runs for 01:27 The video starts with the text 'National Museum of Korea' and video image of outside of the museum. The video goes showing images of outside, lobby, information desk, main hall, the starting part of the museum. Then it shows mains exhibits and people with simple video clips and classic background music. There is no typography, or other expressive techniques. The video is showing static scene of the museum using camera angle changes or 2dimentional edits. Same as the opening scene, the promotional video is closing showing the outside view of the museum from morning to the night time. At the middle part and closing part of the video, Time Remapping effect of Adobe Premiere is applied on the clip. By controlling process speed, the video seems much lively.

\subsection{Promotional yideo $B$}

Promotional video B, promoting MUSEUM KAMPA, has a running time of 02:09. The video starts with pieces of Google map images of the location fly into the screen from the right side, and text shows on the empty space. The video processes using typography to deliver information. The video delivers information by showing video scenes of the museum, people, and objects along with appropriately arranged text. At the end of the video, they shows internet browser to introduce an art project held with Google. The Mouse clicking effect anc browser screen get people excited. The opening scene can be realized using Pemiere Motion effect, which is also used for making an experimental video of this study.

\section{The Expressive Techniques of the Experimental Video}

\subsection{Design Model of the Framework}

Above introducing the new video expressive technique, here is a converging model of AR and culture content. The augmented reality technology, one of the uprising Information \& Communication technologies, opens the door of interaction between the real world and the virtual world. This technology has characteristics of mixing two worlds on an image space, 
connecting objects and users. Using those characteristics, I would like to propose the experimental promotional video model $\mathrm{C}$ for National Museum of Korea, which is a combination of E-museum, and Augmented Reality concepts. The experimental model refers to the concept of Annotation of a research of European Computer-Industry Research Center (ECRC) [11], and the concept of Tracking from Columbia University's research [4]. The experimental video is made using video sources taken at National Museum of Korea, South Korea for the research. The experimental video is made using Motion effects of Adobe Premiere pro program by adjusting Scale, Rotation, Position value for realizing the AR mobile applications effect, and image overlay effect. Image moving effect, which is the main effect of the design model, is mainly applied adjusting Position value of Motion effects.

\subsection{Image Expressive Techniques}

4.2.1. Image Moving Effect using Motion Effect: The experimental video, introducing exhibitions of National Museum of Korea, South Korea, is edited Adobe Premiere pro program. The program suits for copying, pasting, or moving video clips. Also the program is designed to give simple scene change effects or motion effects. Normally, Adobe After Effect is used for editing partial video clips or for applying high-levelvideo effects.However, in this study, I would like to confirm the possibility of applying augmented realistic effects using advanced effects of the Premiere pro program. In detail, user can apply 3D image showing effects, or Image moving effects by adjusting values of Motion effects of Premiere. Let's suppose we are adding an image on a video clip and want to move the image from one point to another point. The very first thing we do is bringing the image file into the timeline. After placing it on the timeline, click the image sitting on the timeline. Then click Effect Controls tap on the top, go to the Motion section under Vided Effects folder. The value we are going to control is 'Position'. Time range can be set by_clicking a clock-shaped figure next to Scale value. Set point 1 on one spot by clicking thê clock-shaped figure. Then adjust Position of the image by changing position value (or moving the image on the right side with a mouse). Set another point, which is poine 2, on another spot in the timeline by clicking the clock-shaped figure again. Adjust Position value fôr the spot, which means the previous adjusted image changes its position as time goes. Other time points, such as spot 3 and spot 4 , can be set up by clicking the button and the image can be adjusted on the time. Whole effects are applied after rendering the edited file and playing it. By adjusting Position value and Scale value together as time processes from one point to other points, image can be seen like moving and changing size at the same time. Also Opacity or Rotation value could be adjusted to present a dynamic image. This image moving effect is useful for composing augmented reality applications concept video scenes. Image or information (Text) mapping enables target users to get information on advanced exhibition contents thought the mobile devices.

4.2.2. Inage Overlay Effect: Image overlay effect is applied on this experimental video by adjusting Opacity-Motion effects. Opacity value has a range of 0 to 100. By increasing the value gradually, image shows up and decreasing the value makes the image disappeared gradually. The opening and closing scene of the video contains this effect, which shows the gradual change of pictures of the museum on the mobile screen. The overlay effect makes it looks like the augmented reality image mapping, using the smartphone, which delivers the massage that user can see images of the other time or in different weather condition.

\section{The Result of the Experimental Model}




\subsection{The Implement Result}

5.1.1. Scene 1: Scene 1 shows how augmented reality applications work. Using mobile devices such as smartphone or tablet, user scans objects with the mobile devices and the augmented reality applications shows additional information of the objects on the screen. The information mapping techniques can be used for actual augmented reality applications in the further. Scene 1 shows the benefits of ICT-culture convergence, that further service for the culture contents are available though the technology and user and the real world interact using the techniques. The overlap techniques are applied using Motion Effects of Premiere pro, and mainly image moving effect and image overlay effect can be applied by adjusting Position, Scale, Opacity value. Specially, the information shown on the screen follows the moving screen that enables the user to explore exhibits freely.

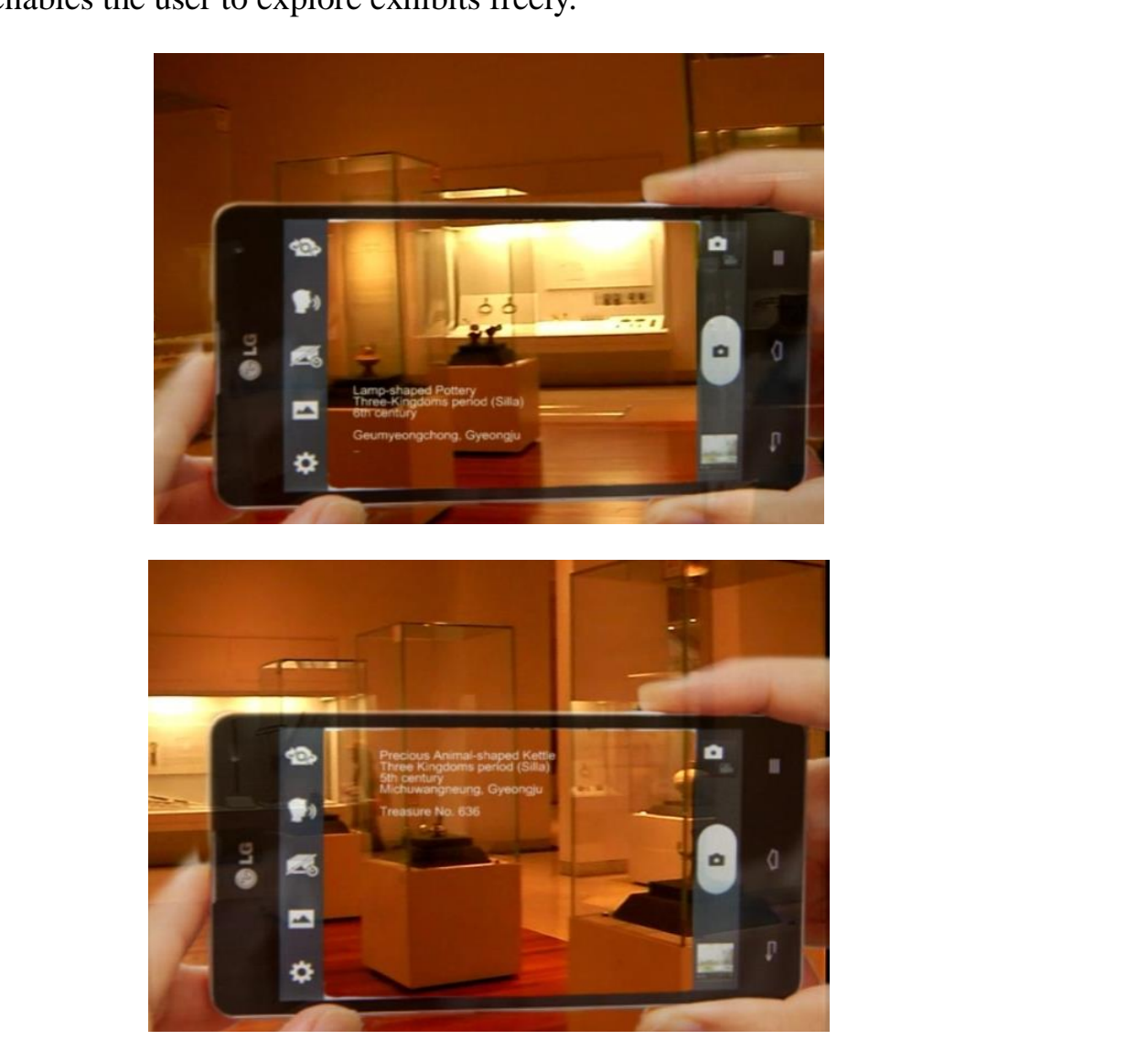

\section{Figure 9. Information Overlay using Augmented Reality}

5.1.2. Scene 2: Scene 2 is made using 3D image pop up techniques based on augmented reality $I n$ this scene, the smart phone features and scans the pagoda, and the 3D image pops up on the screen. Using the augmented feature technique, user gets closer view of entire body or top part of the pagoda. Motion-Video effects-Position value is adjusted from (0.0) to certain point, and size of the 3D image gets bigger by putting higher Scale value. By adjusting the values, the images proceed to front from deep in the screen. This technique can be improved as augmented reality applications that presenting pictures or video of the heritage of the past. 


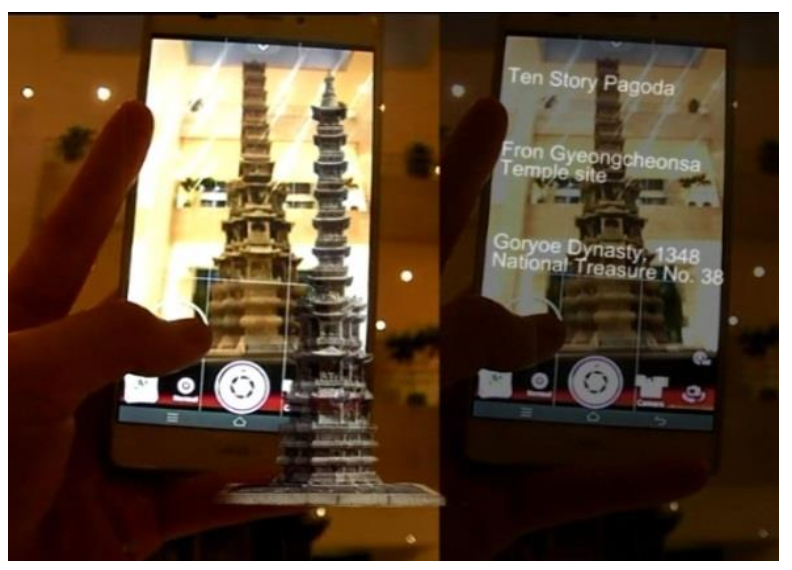

Figure 10. 3D Image Pop Up Using Augmented Reality

5.1.3. Scene 3: Scene 3 features the image overlay effect This effect can be realized by adjusting Dissolve value. However, the effect is applied by adjusting Opacity value under Motion effects section to overlap picture smoothly. The poster images shows up when the smart phone scans the bronze embossed shape, and user sees the 3D poster image on the screen. Considering augmented reality applications are easily scanning fixed shapes, objects or text, scan the embossed shape with mobile device and the poster image pops up on the screen.

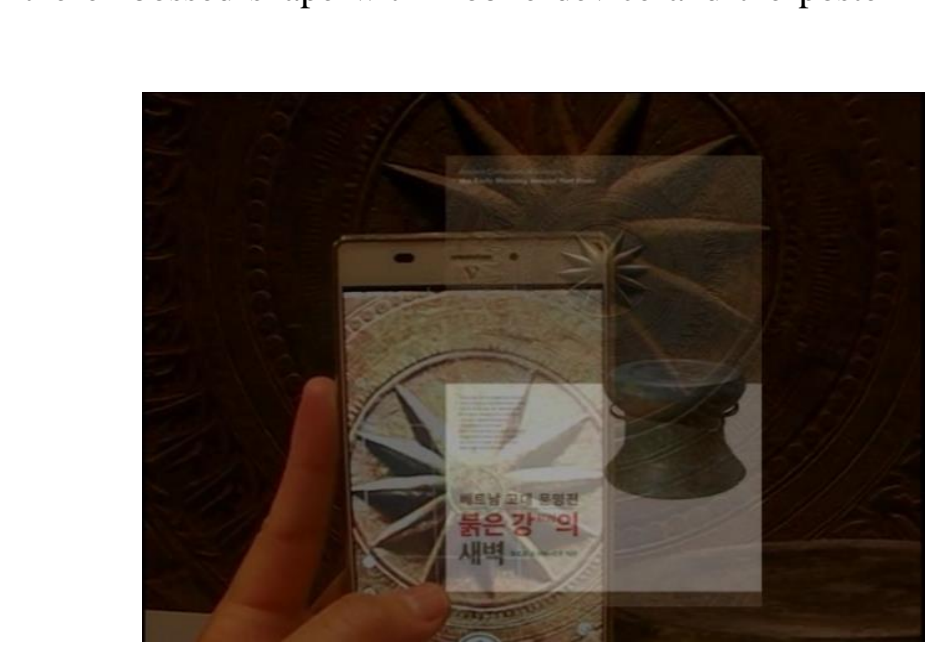

Figure 11. Poster Overlay Effect

\subsection{The Performance Evaluation Analysis}

We've analyzed promotional video A and B in 3.3 Analysis of existing promotional videos, and proposed the experimental promotional video design model based on augmented reality technology and E-museum concept. Video A and video B has running time of 01:27 and 02:09. Source video clips were taken at each museum (National Museum of Korea, and Museum Kampa), and edited. Video A, B and C have similarity of promoting cultural-art organizations, but there are distinctly different expressive techniques. 
Table 2. The Performance Evaluation Analysis Table

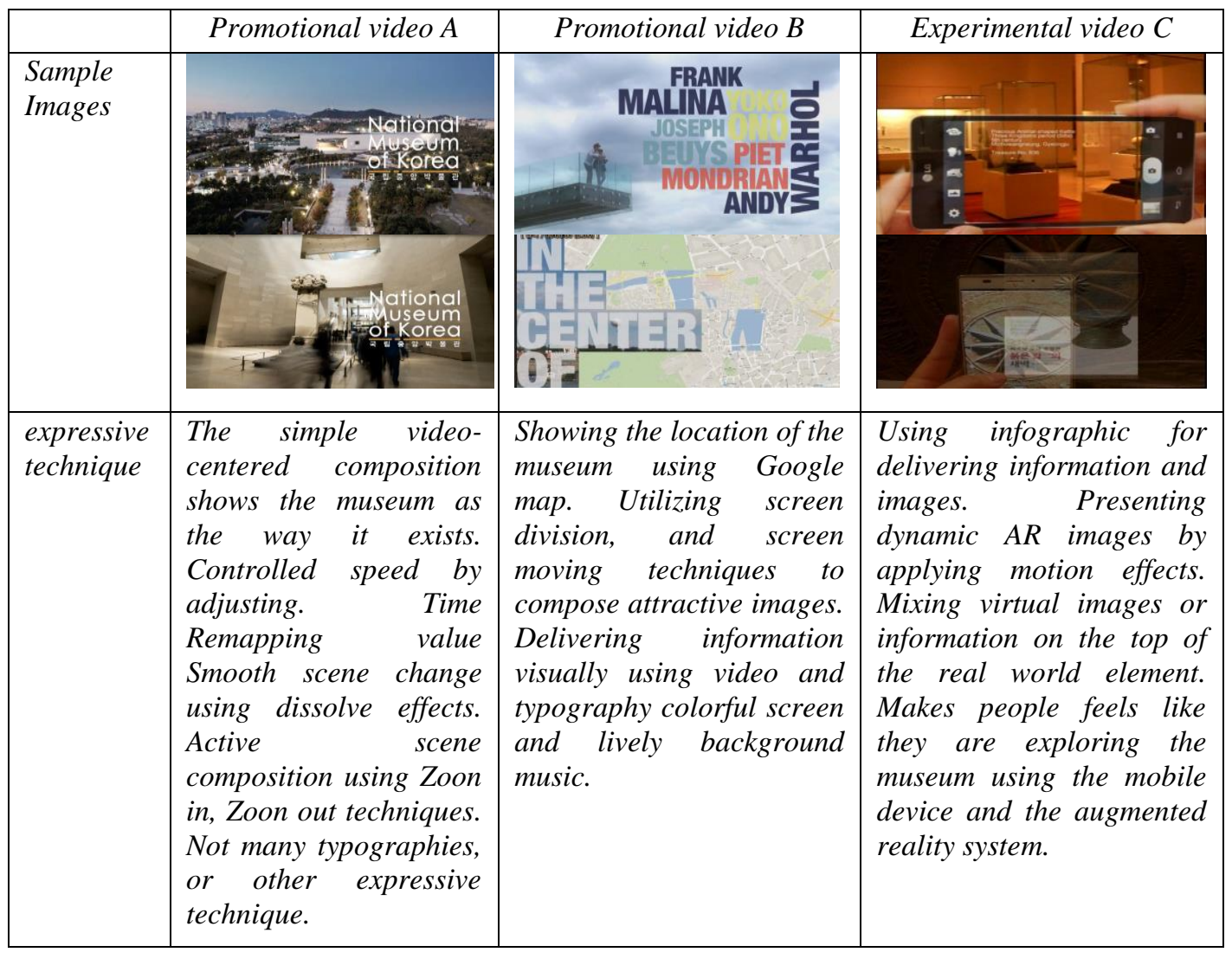

Compare to video A and, video B, which shows the overview of the museum and exhibitions using image angle control and Premiere time remapping techniques, video $\mathrm{C}$ uses advanced expressive techniques such as showing augmented reality concept by Motion effects, or overlap images by contolling Position, and Opacity value. The experimental video uses the virtual exbibition goncept, which enables user interaction with exhibitions. The designed video sets the scenes of exploring the museum using the augmented reality applications, and promotes Dational Museum of Korea by giving 3 dimentional experience to the users There are few technical elements in video A, for example, Text placement, Time remapping or Camera angle changes. Compare to video A, video B contains a little bit of infographic elements in it. On the other hands, video $\mathrm{C}$ contains comparatively diverse of techniques such as infographic, image overlay, image moving and image size changes. In addition, video $\mathrm{C}$ has interesting visual effects such as image overlay or pop-up. Overall, the experimental video show how the future culture industry will be developed as ICT techniques combined in it, and opens the door for possibility to use information \& communication technology for culture contents promotion. In the culture contents field, flat information delivery can not attract consumers. Giving opportunity to interact with the contents is the proper direction of culture contents marketing, which goes with the ICT-culture convergence trend. Therefore the experimental video $\mathrm{C}$ is the new trial to deliver user-centered massage and enable cubical expression.

\subsection{Suggestion of the New Expressive Technique Model}


In this study, I've tried to find the possibility of combining Information \& communication technology with the culture contents industry, specially, for making promotional videos by analyzing one of the uprising ICT technologies, augmented reality, and examining examples of applications in culture contents promotion business. In addition, this study proposed the experimental design model for promotional video. As a result of making the experimental video $\mathrm{C}$, this study confirmed that the new expressive techniques are effectively delivering information to users, and enable communication between the users and the contents. Therefore, this study proposes the new experimental design model for promotional video, and expressive techniques by proposing the experimental video of National Museum of Korea. This model design is differentiated from existing promotional video design on the aspects of maximizing user experience and user interaction. Using the experimental design model, cultural-art organizations expect to attract potential users, and the marketing effects can be spread all over the world through the internet platform such as Youtube or Social petworking service.

\section{Conclusion}

This study proposed the new expressive techniques for making promotional video, which is promoting culture-art organizations, based on the research of Augmented Reality and existing promotional videos. This study referred to some of science papers, and websites of few augmented reality solution companies such as Aurasma and their recent works. After examining augmented reality technology, and suggested the new design model for making promotional video of cultural-art organizations, which is a combination of E-museum, and Augmented Reality concepts. The new experimental model consists of some technical expressive techniques such as $3 \mathrm{D}$ imaging, inage overlay etc, which is distinctly differentiated from existing promotional videos: Overall, the study confirmed that the new experimental techniques enable to deliver culture content effectively, by converging AR and virtual exhibition, 3D imaging concepts and the experience-centered massage of cultural-arts organizations can be effectively and clearlydelivered to the target users.

\section{Acknowledgements}

This work was supported dy a special research grant from Seoul Women's University

\section{References}

[1] T. Starner, S. Mann, B. Rhodes, J. Levine, J. Healey, D. Kirsch and A. Pentland, “Augmented reality through wearable computing", Presence: Teleoperators and Virtual Environments, vol. 6, no. 4, (1997), pp. 386-398.

[2] P. Wellner, W. Mackay and R. Gold, "Computer Augmented Environments: Back to the real world", Communications of the ACM, vol. 36, no. 7, (1993), pp. 24-26.

[3] P. Milgram, H. Takemura, A. Utsumi and F. Kishino, "Augmented reality: A class of displays on the realityintuality continuum. In Photonics for Industrial Applications", International Society for Optics and Photonics, (1995) December, pp. 282-292.

[4] R. T. Azuma, "A survey of augmented reality", Presence: Teleoperators and Virtual Environments, vol. 6, no. 4, (1997), pp. 355-385.

[5] R. Azuma, Y. Baillot, R. Behringer, S. Feiner, S. Julier, and B. MacIntyre, "Recent advances in augmented reality", Computer Graphics and Applications, IEEE, vol. 21, no. 6, (2001), pp. 34-47.

[6] S. Mann, "Mediated reality with implementations for everyday life", Presence Connect, (2002) August, vol. 6.

[7] S. Sanni, "Theory and applications of marker-based augmented reality", Espoo: VTT Technical Research Center of Finland, (2012). 
[8] Virtual Reality \& Augmented Reality Market Forecast by Product (HMD, HUD, Tablet PC, Smartphone) for Gaming, Automotive, Medical, Advertisement, Defense, E-learning \& GPS Applications, Markets and market, (2011) November.

[9] W. E. Mackay, "Augmented reality: linking real and virtual worlds: a new paradigm for interacting with computers", In Proceedings of the working conference on Advanced visual interfaces ACM, (1998) May, pp. $13-21$.

[10] A. Damala, I. Marchal, and P. Houlier, "Merging augmented reality based features in mobile multimedia museum guides", In Proceedings of the XXI International CIPA Symposium, Athens, Greece, (2007) October, pp. 259-264.

[11] E. Rose, D. Breen, K. H. Ahlers, C. Crampton, M. Tuceryan, R. Whitaker, and D. Greer, "Annotating realworld objects using augmented reality. In Computer Graphics: Developments in Virtual Environments (Proceedings of CG International'95 Conference), (1995) June, pp. 357-370.

[12] J.-p. Kim and D.-c. Lee, "Development of Mobile Location Based Service App Using Augmented Reality", Journal of the Korea Institute of Information and Communication Engineering, vol. 18, no. 6, (2014) June, pp. 1481-1487.

\section{Authors}

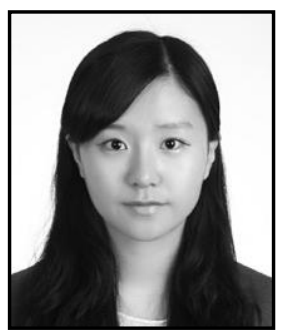

Ga Ram Choi

Seoul Women's University

Department of Contents Desi n

Digital Contents Lab

Researcher

grchoi@dclab.kr

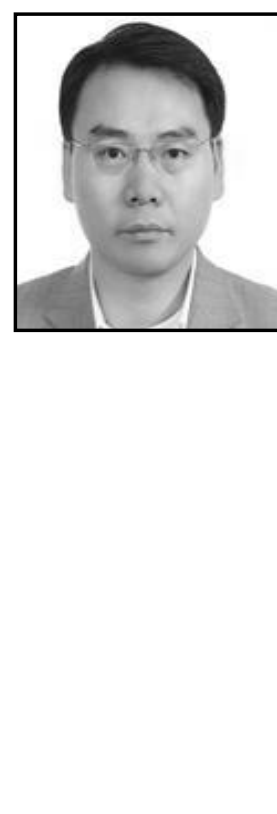

HaK Hyun Choi (Corresponding author)

Korea Uni versity, Ph.D. Course

Seoul Women's University, Professor

Department of Contents Design

Digital Contents Lab

choiidea@gmail.com 